\title{
Recycling of Wash Water as a Way to Reduce the Environmental Load and Increase the Utilization Rate of Raw Materials
}

\author{
T.N. Boitsova ${ }^{1 *}, N . V$. Dementieva $^{1}$ \\ ${ }^{1}$ Far Eastern State Technical Fisheries University, Engineering Disciplines Department, 52 B \\ Lugovaya Str., Vladivostok 690087, Russia
}

\begin{abstract}
The problem of recovering the protein and other valuable components from wash water (low-concentrated suspensions) exists in almost all areas of the food industry. Research in this field is quite successful and is mainly based on the processes of evaporation, flotation, the use of force fields, and other physical and chemical methods. The variety of proposed methods for extracting the protein substances, the permanent search for new methods confirm the incompleteness and urgency of the problem under study. The possibility was demonstrated and the conditions for purification of wash waters of minced meat production and technological liquids for processing the algae were determined. The use of an acidic solution of chitosan was proposed for cleaning the wash water of minced meat production. Considering the ability of chitosan to sediment from solutions having a $\mathrm{pH}$ in the range of 5,6, crystalline sodium bicarbonate was used to adjust the $\mathrm{pH}$ value. The calculation showed that $0,66 \mathrm{~g}$ of sodium bicarbonate was required to neutralize a $2 \%$ solution of chitosan in $2 \%$ acetic acid. The largest mass of the sediment was obtained at the ratio of wash water / chitosan solution as $25 / 2-25 / 4$. Ultrafiltration was used for the purification of technological waters for processing the algae. The results of the study showed that after passing through the filtration bed, the purified water contained 4 times less mineral components and organic substances compared to the original content.
\end{abstract}

\section{Introduction}

Despite the sufficient volumes of catch of aquatic biological resources, regulated by government organizations based on forecasts of research institutes, the volume of catch and the diversity of valuable fish species is constantly decreasing. The search for new raw materials and the development of modern resource-saving processing technologies are the urgent tasks [1,2].

Not all kinds of fish raw materials are easily mechanized, including deep cutting, which allows extracting the maximum amount of valuable food components under production conditions.

\footnotetext{
* Corresponding author: boytsova.tm@dgtru.ru
} 
One of the promising methods of deep cutting of raw materials with maximum accessibility for food use is the technology of producing the food mince [3].

Minced fish, as a semi-finished product, makes it possible to carry out any modifications with nutritional value under production of finished products. It is easy to add any ingredients to the minced meat mass and get a wide range of enriched, functional, specialized, and other kinds of products $[4,5]$.

Minced meat production technology includes the following main processes: cutting the raw materials with the removal of all non-food fragments; grinding the muscle tissue (one or multiple, depending on the required quality); washing the muscle tissue (in the case of the production of washed minced meat); freezing in case of further storage or forming the finished products according to the recipe [6]. The prepared minced meat should not contain non-food fragments or foreign inclusions. The output of minced meat (crushed muscle tissue) is about $20-25 \%$ of the weight of the whole fish. In the case of the production of washed minced meat, the yield is reduced to $12-15 \%$ [7].

Based on the obtained data, one can imagine what kind of load the minced meat production has impact on the ecological system. In the case of cutting the raw materials and grinding into minced meat, this is solid waste, in the case of obtaining the washed minced meat, significant volumes of wash water are also added. According to the requirements of technology and quality characteristics, for the production of one kilogram of washed minced meat (operation of 3-stage washing), from 10 to 15 liters of fresh water is consumed [8].

Washing the muscle tissue of fish is carried out in order to obtain washed minced meat (surimi type), which is used to obtain structured products that mimic various gourmet seafood and not only these ones [9].

Flushing removes most of the sarcoplasmic proteins, hemoglobin, and blood myoglobin, which cause a change in the color of minced meat and catalyze lipid oxidation during storage. Enzymes are removed, including trimethylamine oxidase, which breaks down trimethylamine oxide to formaldehyde, which accelerates the process of protein denaturation [10]. During washing, non-protein nitrogenous substances are removed from the minced meat, which impart an unpleasant odor, dark color to the crushed muscle tissue during storage and promote oxidation, hydrolysis, and denaturation of proteins [11]. The removal of lipids, free fatty acids is partly going on; the bacterial contamination of minced meat is sharply reduced. Metal ions are removed, which prevent the formation of a network-like framework structure in finished products from minced meat during heat treatment [12]. Only myofibrillar proteins should remain in the washed minced meat, which are based on thin actin fibers and thick myosin fibers [11,12].

It should be borne in mind that mainly fresh fish is directed for the production of washed minced meat, and the temperature of the washing water should not exceed $10^{\circ} \mathrm{C}$ to prevent denaturation of proteins of the crushed muscle tissue. Consequently, the eluted components, upset in the wash water, also retain all their native properties. That is, on the one hand, it is a polluting substance, on the other hand, it is a semi-finished product from which valuable substances can be extracted, while reducing the environmental load from production and increasing the utilization rate of raw materials [13].

At the same time, the primary processing of fresh algae provides for their thorough washing and repeated boiling, and dried algae - soaking with simultaneous swelling. In this case, water-soluble salts and organic substances pass into the solution. Losses of organic substances (mannitol, water-soluble colloids, etc.) are undesirable, since they have valuable physiological properties. However, during the primary processing of algae, technological waters (containing important biologically active substances) are formed [14, 15].

The problem of protein extraction from wash water (low-concentrated suspensions) is noted in all areas of the food industry. For this, methods are mainly used based on the 
processes of evaporation, flotation, the use of force fields, and other physical and chemical methods $[16,17]$.

Therefore, under the production of starch, protein substances from potato juice or wash water after separation of starch are transferred into an alkaline solution and salted out by neutralization [18]. Before separation, the wash water or juice are pre-concentrated using the ultrafiltration, and the separation of salted proteins is performed by diafiltration.

In the dairy industry, whey proteins are extracted by acidifying the whey to a $\mathrm{pH}$ of 4,5 5,0 or by alkalizing to a $\mathrm{pH}$ of 7,0 in the presence of a sufficient amount of calcium ions and subsequent heat treatment at a temperature of $60-100 \square \mathrm{C}$. Under this method, 41,15 and $74,06 \mathrm{~g}$ of sediment can be obtained from 1 liter of whey at $\mathrm{pH} 4,5$ and 7,0, respectively, with a total dry matter content of 20,1 and $13,76 \%$, while the protein content in draft is only 9,74 and $6,59 \%$, respectively. However, the effect of high temperatures and the obligatory presence of calcium ions does not allow using this method to extract protein from the wash water of minced meat production.

In other known methods, the isolated protein either loses its native properties, or the process of its extraction requires a large energy consumption, complex preliminary preparation, special equipment, which entails additional economic costs and environmental problems [19].

Thus, the use of flotation methods extends the process in time, which is undesirable in the production of food products, and also requires additional efforts for the final separation of water. Ultrafiltration is carried out only after preliminary separation of washing water from finely ground particles of fish muscle tissue, therefore, as a rule, it should be preceded by flotation, separation or centrifugation. Chemical methods are mainly based on the addition of acids or alkalis and bringing the $\mathrm{pH}$ to unnatural values for food [20].

For the extraction of protein from the washing waters of minced meat production and valuable organic compounds from the technological waters of algae processing, it is important to preserve their native properties, which will make it possible to use the obtained sediment in the production of various kinds of food products, thereby increasing the coefficient of food use of raw materials.

The goal of this research is to study the composition and properties of wash waters for minced meat production and technological waters for processing the algae, to propose methods for extracting the valuable dissolved components and the possibility of their use.

\section{Objects and methods of study}

The objects of study were freshly caught pollock (Treragra chalcogramma), Pacific herring (Clupea pallasii), Japanese kelp (Laminaria japonica), food acid-soluble chitosan.

Determination of the content of protein, fat, dry substances, and minerals was carried out by standard methods.

The amount of fat was determined by the extraction method. The method is based on the extraction of fat from the product with an organic solvent - ether, in a Soxhlet apparatus, evaporation of the solvent and determination of the mass of the extracted fat by weighing.

The determination of the total nitrogen content was carried out by a method based on the oxidation of organic matter by burning it in sulfuric acid in the presence of a catalyst, distilling off the formed ammonia with steam, trapping it with a sulfuric acid solution, and determining the nitrogen content by titration.

Determination of the amount of non-protein nitrogen was implemented by a method based on the separation of non-protein nitrogenous substances from protein ones by precipitation of the latter with trichloroacetic acid and determination of non-protein nitrogen in the filtrate obtained after precipitation by oxidation of organic matter contained in the filtrate when it is burned in concentrated sulfuric acid in the presence of a catalyst, distillation of the resulting 
ammonia with steam, trapping it with a sulfuric acid solution and determining the nitrogen content by back titration.

The determination of the mass fraction of dry substances was carried out by the refractometric method based on the determination of the refractive index of the test solution. An Excellence refractometer (METTLER TOLEDO, Russia) was used for research.

The amount of iodine was determined by a titrometric method based on the formation of a colored complex compound of iodine with sodium nitrate in an acidic medium, and its quantitative determination by titration.

The amount of mineral substances was determined by removing the organic substances from the product by burning in a muffle furnace at a temperature of $500^{\circ} \mathrm{C}$ and determining the ash by weighing.

To purify the technological waters for processing the algae, we used the method of ultrafiltration on a Simplicity® UV apparatus (Chemomed Company, Russia).

The total number of microorganisms (QMAFAnM) was determined by determining the number of grown colonies of microorganisms on nutrient media from agar.

The reliability of the experimental data was ensured by meeting the requirements of mathematical statistics using the standard methods.

To process the results obtained and plot graphical dependencies, the standard programs STATISTICA 13.3 and Microsoft Excel 2016 were used.

\section{Results and Discussion}

It was found that under washing the muscle tissue of cut fish (thoroughly cleaned fillet or carcass), finely crushed particles of crushed tissue leave in the wash water, in addition to water-soluble proteins, lipids, and extractive substances. The composition of the wash water depends on the type of raw material, the degree of crushing of muscle tissue before washing, the volume of water and the number of washes [10-12].

The most rational degree of grinding the muscle tissue of fish before washing is considered to be the size of particles 3 - $4 \mathrm{~mm}$ [11]. A finer grinding leads to greater losses of raw materials during washing, a larger one - to a larger volume of water for washing, an increase in the duration of washing and excessive swelling of meat, a decrease in the quality of minced meat [12].

It is known that under the minced meat washing cycle with stirring, the amount of extracted protein increases during the first 9 minutes, then the process stabilizes, therefore the total number of washes should be at least three, with a total contact time of the fish muscle tissue with water of at least 30 minutes [10-12].

The composition of the wash water under washing the crushed muscle tissue from fresh fish with predominantly light and predominantly dark muscle tissue (pollock and herring), after washing three times in a ratio of 1 / 3 (muscle tissue / water), is approximately the same (Table 1).

Table 1. Technochemical characteristics of wash waters formed during the production of washed fish mince.

\begin{tabular}{|c|c|c|c|c|c|}
\hline \multirow[b]{2}{*}{ Kind of fish } & \multicolumn{4}{|c|}{ Mass fraction, $\mathrm{g} / \mathrm{L}$} & \multirow{2}{*}{$\begin{array}{c}\text { QMAFAn } \\
\text { M, } \\
\text { COE / g }\end{array}$} \\
\hline & protein & lipids & $N_{n p} \cdot 10^{-4}$ & Dry matter & \\
\hline Pollock & 1,80 & 0,42 & 0,29 & 15,05 & $2 \cdot 10^{2}$ \\
\hline
\end{tabular}




\begin{tabular}{|l|l|l|l|l|l|}
\hline $\begin{array}{l}\text { Pacific } \\
\text { herring }\end{array}$ & 2,53 & 1,89 & 0,35 & 20,12 & $5 \cdot 10^{3}$ \\
\hline
\end{tabular}

The slightly increased content of extractive substances in the water from washing the crushed muscle tissue of herring is explained by the peculiarities of its morphological structure and chemical composition. Considering that almost pure muscle tissue is subjected to grinding and washing, after removing all non-food inclusions, the washing water contains only extractive substances of fish muscle tissue, the amount of which ranges from 10 to 50 $\mathrm{g} / \mathrm{L}$ (on average, 15 and $20 \mathrm{~g} / \mathrm{L}$ for pollock and Pacific herring, respectively).

In addition, in the wash water there are also finely disintegrated fragments of muscle tissue, which passed through specialized devices under separating the washed minced meat from the wash water. In this case, the larger fragments settle rather quickly, the smaller ones are in suspension.

From the standpoint of the environmental load, it is polluting wash water; from the standpoint of the integrated use of raw materials, it is a full-fledged raw material for obtaining an additional amount of products.

Under processing the algae, it was found that the rate of extraction of mineral and organic substances was directly dependent on the temperature of the water, the duration of the process, the degree of grinding, the intensity of mixing, the magnitude of the hydromodule, and the frequency of water change. The amount of extracted substances in the technological waters formed during the processing of Japanese kelp by cooking in water at a temperature of $90^{\circ} \mathrm{C}$ is in Table 2 .

Table 2. The amount of extracted substances in the technological waters formed during the processing of Japanese kelp.

\begin{tabular}{|c|c|c|c|c|c|}
\hline $\begin{array}{c}\text { Duration of } \\
\text { extraction, min }\end{array}$ & Salt & Iodine & $\begin{array}{c}\text { Organic } \\
\text { matter }\end{array}$ & $\begin{array}{c}\text { QMAFAnM, } \\
\text { COE / g }\end{array}$ & \\
\hline 5 & 15,0 & 10,2 & 2,3 & $2 \cdot 10^{1}$ & \\
\hline 10 & 23,6 & 21,4 & 5,4 & $5 \cdot 10^{1}$ & \\
\hline 20 & 50,0 & 46,4 & 9,7 & $1 \cdot 10^{2}$ & \\
\hline 30 & 56,8 & 54,3 & 14,0 & $3 \cdot 10^{2}$ & \\
\hline 40 & 66,7 & 69,3 & 26,4 & $8 \cdot 10^{2}$ & \\
\hline
\end{tabular}

The total microbial count (QMAFAnM) gradually increases, with the accumulation of mineral and organic substances in the wash waters and varies from $2 \cdot 10^{1}$ to $8 \cdot 10^{2}$.

One of the promising methods for extracting the isolated valuable components from rinsing waters is the use of complexing agents that are allowed for food use. A water-soluble chitosan approved for food use was selected for research [21].

Chitosan is a product of processing the chitin-containing wastes of crustaceans, which, by chemical nature, has polyelectrolytic properties, while its amino groups provide a strong covalent bond with protein substances $[21,22]$.

Chitin and chitosan are formed and decomposed by living organisms, in natural conditions they break down into simple sugar and glucosamine [21], therefore it is safe for humans, animals and the environment, in many respects meets the requirements for sorbents. The reactivity of chitosan is due to the presence of free amino groups in its molecules, it is non-toxic, compatible with food components, decomposes under the action of enzymes, and has a beneficial effect on lipid, protein, and vitamin metabolism [21-23].

The water-soluble protein of the wash water is in the native state, because under washing the crushed muscle tissue of fish, the water temperature is strictly controlled and should not 
exceed $10-12^{\circ} \mathrm{C}$ in order to avoid denaturation and deterioration of the functional properties of minced meat [11].

When studying the possibility of precipitation of a water-soluble protein, dry chitosan was used. It was added to the wash water obtained in the production of washed pollock minced meat in an amount of $2-10 \%$ with an interval of 2 . The chitosan flakes immediately sank to the bottom, and after 10-15 min their swelling and the formation of an ash-like sediment mass were observed.

After filtering and drying the sediment, it turned out that its mass was practically equal to the mass of added chitosan with a small amount of finely dispersed muscle particles sedimened by gravity that were present in the wash water. Obviously, there was simply swelling of chitosan in water, and not its interaction with dissolved particles [23].

The activity of the functional groups of chitosan increases when it is converted into a salt form [24]; therefore, a $2 \%$ solution of chitosan in $2 \%$ acetic acid was prepared for the study, the solution was kept for at least 2 hours until the complete dissolution of chitosan.

The functional groups of water-soluble protein and chitosan molecules interact with each other and form aggregate complexes, but taking into account the ability of chitosan to sediment from solutions with a $\mathrm{pH}$ in the region of $5,6[25,26]$, these complexes can be converted into a sediment.

Crystalline sodium bicarbonate was used to adjust the $\mathrm{pH}$. The calculation showed that $0,66 \mathrm{~g}$ of sodium bicarbonate is required to neutralize a $2 \%$ solution of chitosan in $2 \%$ acetic acid. Taking into account that the process of neutralization of chitosan can be influenced by the active chemical compounds of the wash water, the actual required amount was determined. For the study, a solution of chitosan $(100 \mathrm{ml})$ was added from 0,60 to $1,65 \mathrm{~g}$ of sodium bicarbonate, stirred, taken in $20 \mathrm{ml}$ and combined with $50 \mathrm{ml}$ of wash water; the mixture was left to form a sediment, then centrifuged and dried.

Depending on the amount of sodium bicarbonate introduced, the amount of the formed sediment changed. The largest mass of the sediment was noted with the addition of $1,2 \mathrm{~g}$ of $\mathrm{NaHCO}_{3}$, that is, the amount of sodium bicarbonate actually required to neutralize chitosan is almost 2 times higher than the calculated one (Table 3 ).

Table 3. The mass of the released protein-chitosan sediment, depending on the mass of sodium bicarbonate, which was used for neutralization.

\begin{tabular}{|c|c|c|c|c|c|c|c|c|}
\hline \multirow{2}{*}{ Component } & \multicolumn{9}{|c|}{ Mass, g } \\
\cline { 2 - 9 } & $\mathbf{1}$ & $\mathbf{2}$ & $\mathbf{3}$ & $\mathbf{4}$ & $\mathbf{5}$ & $\mathbf{6}$ & $\mathbf{7}$ & $\mathbf{8}$ \\
\hline $\mathrm{NaHCO}_{3}$ & 0,60 & 0,75 & 0,90 & 1,05 & 1,20 & 1,35 & 1,50 & 1,65 \\
\hline Sediment & - & - & - & 0,4848 & 0,9773 & 0,7071 & 0,8846 & 0,5953 \\
\hline
\end{tabular}

The duration of the exposure for the formation of sediment after neutralization was 7 - 15 minutes.

The largest mass of sediment was obtained with the ratio of wash water / chitosan solution as $25 / 2-25 / 4$ (Figure 1). 


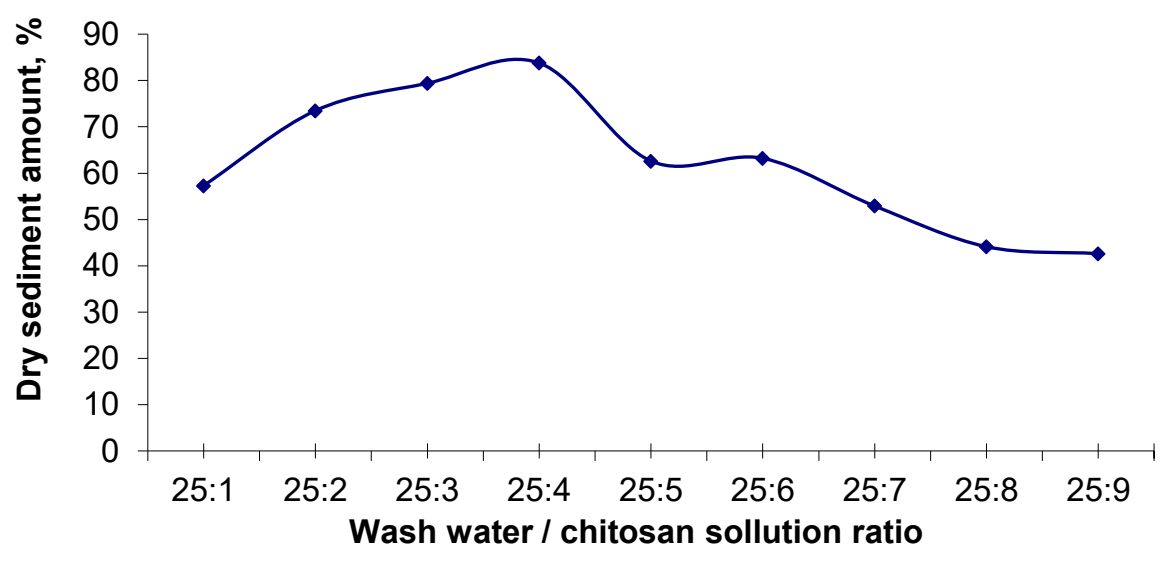

Fig.1. Sediment yield depending on the ratio of wash water / chitosan solution

Fish with a high content of dark muscles can be directed to the production of washed minced fish, so the natural $\mathrm{pH}$ values of the wash water can range from 5 to 7 . This can affect the process of isolating the protein substances from the wash water. Therefore, a neutralized solution of chitosan to $\mathrm{pH} 6,1-6,4$ was added to wash water (previously adjusted with sodium bicarbonate to $\mathrm{pH} 5-7$ ), mixed thoroughly, left to form a sediment for $15 \mathrm{~min}$, and centrifuged.

The resulting sediment is a dense mass corresponding to the organoleptic characteristics (color, taste, and smell) of unwashed minced fish. The consistency is from smudged to curdled. The supernatant liquid is clear or slightly turbid compared to the wash water. The amount of protein that passed into the sediment from the wash water, determined by the difference between the protein content in the original wash water and in the water after separation of the sediment, was $78-93 \%$ (Table 4). The best results were obtained when the $\mathrm{pH}$ of the wash water was between 6 and 7, the water remaining after centrifugation was almost transparent.

Table 4. Influence of the $\mathrm{pH}$ of the wash water on the amount of precipitate isolated.

\begin{tabular}{|c|c|c|}
\hline $\begin{array}{c}\text { pH of the initial wash } \\
\text { water }\end{array}$ & $\begin{array}{c}\text { Sediment mass, } \\
\mathbf{g}\end{array}$ & $\begin{array}{c}\text { The amount of protein that has } \\
\text { passed into the sediment, \% }\end{array}$ \\
\hline 5,00 & 0,0697 & 88,0 \\
\hline 5,64 & 0,0738 & 89,9 \\
\hline 5,92 & 0,0882 & 90,5 \\
\hline 6,59 & 0,0927 & 93,0 \\
\hline 6,85 & 0,0905 & 92,8 \\
\hline 7,00 & 0,0897 & 91,5 \\
\hline
\end{tabular}

The mass of the formed sediment depends on the $\mathrm{pH}$ value of the mixture wash water chitosan solution, after adding neutralized chitosan in the range of 6,6-7,1 (Figure 2). Acceptable conditions for protein precipitation are $\mathrm{pH}$ values of the mixture in the range of $6,8-7,1$ (Figure 3). 


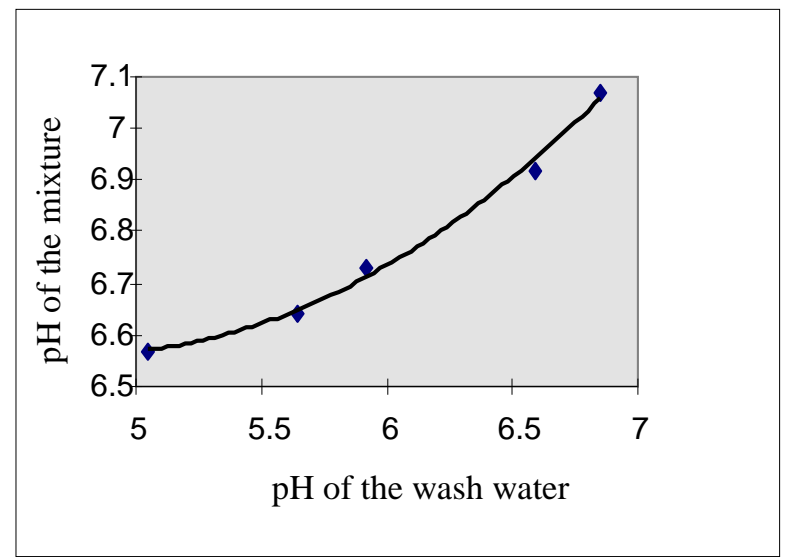

Fig. 2. The $\mathrm{pH}$ values of the mixture wash water - chitosan solution with a change in the $\mathrm{pH}$ of the wash water.

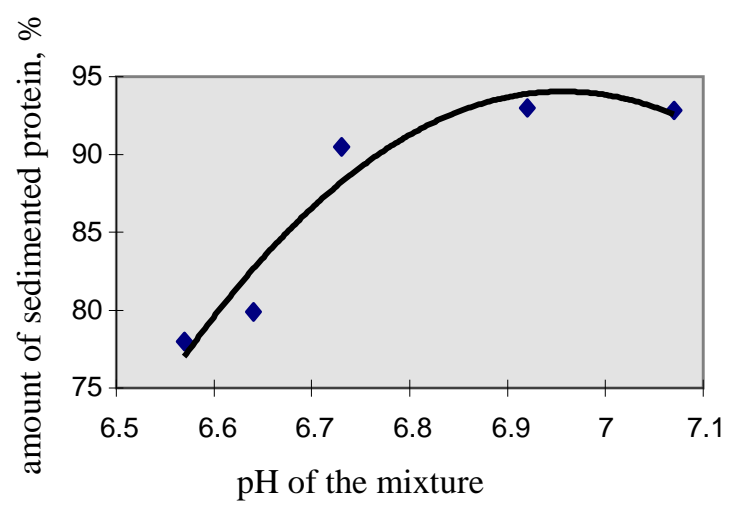

Fig. 3. The amount of sedimented protein with a change in the $\mathrm{pH}$ values of the mixture wash water chitosan solution

The largest amount of sediment also corresponds to a larger amount of protein that has passed into the sediment. At all the studied $\mathrm{pH}$ values of the wash waters, sedimentation occurs, while the best results were obtained at $\mathrm{pH} 6,59-6,85$, which corresponds to the natural $\mathrm{pH}$ values of cod fish species, which are most often used for the production of washed minced fish.

Under purifying the technological waters for processing the algae, we used the method of ultrafiltration on a Simplicity® UV apparatus (Chemomed Company, Russia).

The results of the study demonstrated that after passing through the filtration bed, the purified water contained 4 times less mineral components and organic substances in comparison with the original content.

\section{Conclusions}

In further research, the possibility of using the protein-chitosan sediment obtained after cleaning the wash water of minced meat production and the concentrated substrate of mineral and organic substances after cleaning the technological waters of processing the algae to increase the coefficient of the integrated use of raw materials will be studied. 
Thus, the possibility has been demonstrated and the conditions have been determined for the purification of wash waters of minced meat production and technological liquids for processing the algae. Treated water can be directed for secondary use in technological processes or as a technical liquid for industrial needs. In addition, the discharge of these waters as production waste will not pose an ecological threat to the environment.

\section{References}

1. A. Y. Volkov, Donskova, L.A., Kotkova, V.V. Technological solutions in food production in the context of ensuring their quality and safety. New technologies, 3, 2027 (2018)

2. O.V. Chernyshova, Tsibizova, M.E. Technochemical composition and functional and technological properties of underutilized fish raw materials of the Volga-Caspian basin. Bulletin of the ASTU, Ser. Fisheries, 189-194 (2012)

3. C. James, Purnell, G., James, S.J. A Review of Novel and Innovative Food Freezing Technologies. Food and Bioprocess Technology, 8 (8), 1616-1634 (2014) DOI: http://dx.doi.org/10.1007/s11947-015-1542-8

4. L.V. Antipov, Voronkova, Yu.V. Recipe development of minced meat using the ECOLIGHT NATIVE dietary fibers. Bulletin of the Voronezh State University of Engineering Technologies, 4,116-119 (2013)

5. I.S. Drozdetskaya, Berezovikova, I.P. The influence of liquid smoke flavoring on the rheological characteristics of minced fish. Bulletin of the Voronezh State University of Engineering Technologies, 80 (1), 193-198 (2018) DOI: https://doi.org/10.20914/23101202-2018-1-193-198.

6. S. Hasharifian, Ebrahim, A., Mortazavi, M., Moghadam, M. Effects of refrigerated storage on the microstructure and quality of Grouper (Epinephelus coioides) fillets. Journal Food Science Technology, 51(5), 929-935 (2014)

7. L.I. Drozdova, Pivnenko, T.N. Features of the rheological indicators of minced meat from deep-sea fish and products from them. Izvestiya of TINRO, 172, 274-281 (2013)

8. D. Mozaffarian, Rimm, E. B. Fish intake, contaminants, and human health - Evaluating the risks and the benefits. Journal of the American Medical Association, 296, 1885-1899 (2006): DOI: https://doi.org/10.1001/jama.296.15.1885.

9. F. Zhang, Fang, L., Wang, C., Shi, L. et al. Effects of starches on the textural, rheological, and color properties of surimi-beef gels with microbial tranglutaminase. Meat Science, $93 \quad$ (3), 533-537 (2013) DOI: https://doi.org/ 10.1016/j.meatsci.2012.11.013.

10. M. D. Mukatova, Kirichko, N. A. Romanenkova, E. N., Zotova N. Yu. Development of a technology for the production of washed minced fish from unprofitable objects of the Volga-Caspian basin. Bulletin of the Murmansk State Technical University, 19 (3), 625632 (2016) DOI: 10.21443/1560-9278-2016-3-625-632

11. N.V. Yartseva, Dolganova, N.V. Study of the possibility of improving the quality of minced fish by washing with organic acids. ASTU Bulletin. Ser. Fishery, 1, 158-165 (2011)

12. E.N. Golikova, Mukatova, M.D., Kirichko, N.A. Study of the possibility of producing the minced meat of the "surimi" type from underutilized small biological resources of the Volga-Caspian region. ASTU Bulletin. Ser. Fishery, 1, 103-109 (2011)

13. A.S. Vinnov, Mayevskaya, T.N., Dolganova, N.V. Influence of the type of wash water on the rheological properties of gels from surimi minced meat. ASTU Bulletin. Ser. Fishery, 1, 180-189 (2013) 
14. R.R. Kabirov, Gaisina, L.A., Sukhanova, N.V., Krasnova, V.V. Biotechnological aspects of the use of microscopic algae and cyanobacteria. International Journal of Experimental Education, 7, 128-129 (2016)

15. T.I. Vishnevskaya, Aminina, N.M., Guruleva, O.N. Development of technology for producing the iodine-containing products from Laminaria japonica. TINRO Bulletin, 129, 163-169 (2001)

16. V.N. Yaromsky, Wastewater treatment for food and processing enterprises. Minsk: BSU Publishing Center, 171 (2009)

17. V.V. Ilyushin, Modern methods of wastewater treatment. Modern techniques and technologies, 2, 47-54 (2017)

18. I.S. Zaitseva, Zaitseva, N.A., Voronina, A.S. Methods for intensifying the biological wastewater treatment in aeration tanks. Bulletin of the Kuzbass State Technical University, 2, 90-91 (2010)

19. E.S. Gogina, Kulakov, A.A. Development of technology for modernization of artificial biological wastewater treatment facilities. MSSU Bulletin, 11, 204-209 (2012)

20. N.N. Zhurkin, Alibekov, S.Ya. Improvement of mechanical wastewater treatment. Bulletin of the Volga State Technological University. Series: Forest. Ecology. Nature management, 1 (17), 92-97 (2013)

21. V.E. Kamskaya, Chitosan: structure, properties and use. Scientific Review. Biological sciences, 6, 36-42 (2016)

22. V.M. Bykova, Nemtsev, S.V. Raw materials sources and methods for producing the chitin and chitosan. Chitin and Chitosan. Production, properties and, application, 7-10 (2002)

23. L.F. Gorovoy, Kosyakov, V.N. Sorption properties of chitin and its derivatives: Chitin, its structure and properties. Chitin and Chitosan. Production, properties and application. 217-246 (2002)

24. A.I. Albulov, Frolova, M.A., Muradyan, Zh.Yu. Chitosan as a new natural enterosorbent for veterinary medicine and animal husbandry. All-Russian Research and Technological Institute of Biological Industry. P.6-8 (2007)

25. P. M. De la Torre, Torrado, S. Interpolymer complexes of polyacrylic acid. Biomaterials. 24(8), 1459-1468 (2003)

26. Song Peng-fei, Wang Rong-min, Wang Yun-pu. Preparation and properties of hydrogels based on chitosan and polyvinylpyrrolidone, sensitive to $\mathrm{pH}$ changes. J. Northw. Norm. Univ. Natur.Sci., 40, 53-55 (2004) 